Korean J. Math. 21 (2013), No. 3, pp. 325-330

http://dx.doi.org/10.11568/kjm.2013.21.3.325

\title{
TROTTER-KATO THEOREM IN THE WEAK TOPOLOGY
}

\author{
Young SeOp LeE
}

\begin{abstract}
In this paper, we prove Trotter-Kato theorem in the weak topology if $X^{*}$ is a uniformly convex Banach space.
\end{abstract}

\section{Introduction}

Let $X$ be a Banach space. A family $\{T(t): t \geq 0\}$ of bounded linear operators from $X$ into itself is called a contraction $C_{0}$ semigroup on $X$ if $T(0)=I, T(t+s)=T(t) T(s)$ for $t, s \geq 0$, for each $x \in X T(t) x$ is continuous in $t \geq 0$ and $\|T(t) x\| \leq\|x\|$ for $t \geq 0$ and $x \in X$.

The linear operator $A$, defined by

$$
A x=\lim _{h \rightarrow 0} \frac{1}{h}(T(h) x-x)
$$

for $x \in D(A)=\left\{x \in X: \lim _{h \rightarrow 0}(T(h) x-x) / h\right.$ exists $\}$, is called the generator of a contraction $C_{0}$ semigroup $\{T(t): t \geq 0\}$ and $D(A)$ is the domain of $A$.

The resolvent set of $A$ is denoted by $\rho(A)$ and for $\lambda \in \rho(A) R(\lambda, A)=$ $(\lambda I-A)^{-1}$ is the resolvent operator of $A$, and we define the Yosida approximation of $A$ by

$$
A_{\lambda}=\lambda A R(\lambda, A)=\lambda^{2} R(\lambda, A)-\lambda I .
$$

Received July 31, 2013. Revised September 3, 2013. Accepted September 3, 2013. 2010 Mathematics Subject Classification: 47D06, 34K07.

Key words and phrases: Trotter-Kato theorem, weak topology, uniformly convex Banach spaces.

This work was supported by a research grant from Seoul Women's Univer$\operatorname{sity}(2012)$.

(c) The Kangwon-Kyungki Mathematical Society, 2013.

This is an Open Access article distributed under the terms of the Creative commons Attribution Non-Commercial License (http://creativecommons.org/licenses/by -nc/3.0/) which permits unrestricted non-commercial use, distribution and reproduction in any medium, provided the original work is properly cited. 
For more information about $C_{0}$ semigroups and their generators, we refer $[2,5]$.

The object of this paper is to discuss when the Trotter-Kato approximation theorem holds for the weak operator topology. This type of result plays a tool for the numerical study of partial and stochastic (partial) differential equations and is one of methods to use study a complicated operator. For the strong operator topology, the convergence of a sequence of $C_{0}$ semigroups $\left\{T_{n}(t): t \geq 0\right\}$ is related to the convergence of their generators $A_{n}$ and their resolvents $R\left(\lambda, A_{n}\right)$. Replacing the strong convergence of the resolvents by the weak convergence does not imply the weak convergence of corresponding $C_{0}$ semigroups, while the inverse is true by the Laplace transform representation of the resolvent of the generator and Lebesgue's theorem. In [1], the weak convergence of generators or resolvents of their generators does not imply the weak convergence of $C_{0}$ semigroups even if the generators are bounded.

In [3], G. Marinoschi has proved that the weak version of Trotter-Kato approximation theorem with some restrictions on generators is valid for a Hilbert space. In this paper we extend this result to a Banach space $X$ whose dual space $X^{*}$ is uniformly convex for contraction $C_{0}$ semigroups.

With the uniform convexity of $X^{*}$ we have the uniform continuity of the dual mapping. The inner product of a Hilbert space is replaced by the dual mapping and the uniform continuity of the dual mapping is essential for the proof of our main result.

\section{Weak Convergence}

Let $X$ be a Banach space and let $X^{*}$ be its dual space. We denote the value $x^{*}(x)$ of $x^{*} \in X^{*}$ at $x \in X$ by the duality pairing $\left\langle x, x^{*}>\right.$ or $<x^{*}, x>$.

We recall that for each $x \in X$ the dual mapping $J: X \rightarrow X^{*}$ is defined by

$$
J(x)=\left\{x^{*} \in X^{*}:<x, x^{*}>=\|x\|^{2}=\left\|x^{*}\right\|^{2}\right\} .
$$

Note that $J(x)$ is a subset of $X^{*}$ and $J(x) \neq \emptyset$ for all $x \in X$, by HahnBanach Theorem. Hence $J$ can be viewed as a multi-valued function. Under some restrictions on $X^{*}, J$ can be a single-valued. If $X$ is a Hilbert space, then $J$ is the identity mapping on $X$. With the uniform convexity of $X^{*}$, we have the following properties of the dual mapping 
(see [4]). For example, Hilbert spaces are uniformly convex and $L^{p}$ spaces $(1<p<\infty)$ are also uniformly convex.

THEOREM 1. If $X^{*}$ is a uniformly convex Banach space, then the dual mapping $J$ is single-valued and uniformly continuous on every bounded subsets of $X$.

Theorem 2. If $u:(a, b) \rightarrow X$ has a weak derivative $u^{\prime}(t)$ and $\|u(t)\|$ is differentiable, then

$$
\frac{d}{d t}\|u(t)\|^{2}=2<u^{\prime}(t), f>\text { for } f \in J(u(t)) .
$$

We set $w-\lim$ by the weak limit and the Yosida approximation of $A_{n}$ by $A_{n, \lambda}$ for any $\lambda>0$.

Theorem 3. Let $X$ be a Banach space whose dual space $X^{*}$ is uniformly convex. Let $\left\{T_{n}(t): t \geq 0\right\}$ be a sequence of contraction $C_{0}$ semigroups with generators $A_{n}$ and let $\{T(t): t \geq 0\}$ be a contraction $C_{0}$ semigroup with generator $A$. Suppose that

$$
w-\lim _{n \rightarrow \infty} R\left(\lambda, A_{n}\right)^{k} x=R(\lambda, A)^{k} x, \quad k=1,2, \cdots
$$

for $x \in X$ and

$$
D=\left\{x \in \cap_{n=1}^{\infty} D\left(A_{n}\right): \sup _{n \geq 1}\left\|A_{n} x\right\|<\infty\right\}
$$

is dense in $X$. Then

$$
w-\lim _{n \rightarrow \infty} T_{n}(t) x=T(t) x
$$

for all $x \in X$ and the convergence is uniform on bounded $t$ - intervals.

Proof. Let $0 \leq t \leq T$ and $x \in D$. Then

$$
\begin{aligned}
& \left|<T_{n}(t) x-T(t) x, \phi>\right| \\
& \quad \leq \quad\left|<T_{n}(t) x-e^{t A_{n, \lambda}} x, \phi>\right|+\left|<e^{t A_{n, \lambda}} x-e^{t A_{\lambda}} x, \phi>\right| \\
& \quad+\left|<e^{t A_{\lambda}} x-T(t) x, \phi>\right|
\end{aligned}
$$

for each $\phi \in X^{*}$.

By Hille-Yosida's Theorem, we have

$$
\lim _{\lambda \rightarrow \infty}\left\|e^{t A_{\lambda}} x-T(t) x\right\|=0 \text { and } \lim _{\lambda \rightarrow \infty}\left\|e^{t A_{n, \lambda}} x-T_{n}(t) x\right\|=0,
$$

uniformly on bounded $t$-intervals. Hence we have

$$
\lim _{\lambda \rightarrow \infty}<T_{n}(t) x-e^{t A_{n, \lambda}} x, \phi>=0 \text { and } \lim _{\lambda \rightarrow \infty}<T(t) x-e^{t A_{\lambda}} x, \phi>=0,
$$


uniformly on $[0, T]$ for $x \in D$ and $\phi \in X^{*}$.

We will show that the convergence $\lim _{\lambda \rightarrow \infty}<T_{n}(t) x-e^{t A_{n, \lambda}} x, \phi>=0$ is uniform with respect to $n$.

Let $u_{n, \lambda}(t)=e^{t A_{n, \lambda}} x$ and $u_{n}(t)=T_{n}(t) x$. Then we have the following properties which are given in the proof of Hille-Yosida's theorem.

$$
\begin{aligned}
& \frac{d}{d t} u_{n, \lambda}(t)=A_{n, \lambda} u_{n, \lambda}(t), \quad u_{n, \lambda}(0)=x \\
& \frac{d}{d t} u_{n}(t)=A_{n} u_{n}(t), \quad u_{n}(0)=x \\
& \left\|u_{n, \lambda}(t)\right\| \leq\|x\| \\
& \left\|\frac{d}{d t} u_{n, \lambda}(t)\right\|=\left\|A_{n, \lambda} u_{n, \lambda}(t)\right\| \leq\left\|A_{n, \lambda} x\right\| \\
& \left\|R\left(\lambda, A_{n}\right) x\right\| \leq \frac{1}{\lambda}\|x\| \\
& <A_{n, \lambda} x, J(x)>\leq 0 \text { for } x \in X \\
& \left\|A_{n, \lambda} x\right\|=\left\|\lambda A_{n} R\left(\lambda, A_{n}\right) x\right\| \leq\left\|A_{n} x\right\| \text { for } x \in D\left(A_{n}\right)
\end{aligned}
$$

By Theorem 2, we have

$\frac{1}{2} \frac{d}{d t}\left\|u_{n, \lambda}(t)-u_{n, \mu}(t)\right\|^{2}=<A_{n, \lambda} u_{n, \lambda}(t)-A_{n, \mu} u_{n, \mu}(t), J\left(u_{n, \lambda}(t)-u_{n, \mu}(t)\right)>$ for $\lambda, \mu>0$.

Consider the following estimates.

$$
\begin{aligned}
\|\left(u_{n, \lambda}(t)-\lambda R\left(\lambda, A_{n}\right) u_{n, \lambda}(t)-\left(u_{n, \mu}(t)-\mu R\left(\mu, A_{n}\right) u_{n, \mu}(t)\right) \|\right. \\
=\|-\frac{1}{\lambda}\left(\lambda^{2} R\left(\lambda, A_{n}\right) u_{n, \lambda}(t)-\lambda u_{n, \lambda}(t)\right) \\
\quad+\frac{1}{\mu}\left(\mu^{2} R\left(\mu, A_{n}\right) u_{n, \mu}(t)-\mu u_{n, \mu}(t)\right) \| \\
=\quad\left\|-\frac{1}{\lambda} A_{n, \lambda} u_{n, \lambda}(t)+\frac{1}{\mu} A_{n, \mu} u_{n, \mu}(t)\right\| \\
\leq \frac{1}{\lambda}\left\|A_{n, \lambda} u_{n, \lambda}(t)\right\|+\frac{1}{\mu}\left\|A_{n, \mu} u_{n, \mu}(t)\right\| \\
\leq \quad\left(\frac{1}{\lambda}+\frac{1}{\mu}\right)\left\|A_{n} x\right\| .
\end{aligned}
$$

Let $\varepsilon>0$ be given. Since $x \in D$ and $J$ is uniform continuous,

$$
\left\|J\left(u_{n, \lambda}(t)-u_{n, \mu}(t)\right)-J\left(\lambda R\left(\lambda, A_{n}\right) u_{n, \lambda}(t)-\mu R\left(\mu, A_{n}\right) u_{n, \mu}(t)\right)\right\|<\varepsilon
$$

for sufficiently large $\lambda$ and $\mu$. 
By the uniform continuity of $J$, we have

$$
\begin{aligned}
< & A_{n, \lambda} u_{n, \lambda}(t)-A_{n, \mu} u_{n, \mu}(t), J\left(u_{n, \lambda}(t)-u_{n, \mu}(t)\right)> \\
= & <A_{n, \lambda} u_{n, \lambda}(t)-A_{n, \mu} u_{n, \mu}(t), J\left(u_{n, \lambda}(t)-u_{n, \mu}(t)\right) \\
& \quad-J\left(\lambda R\left(\lambda, A_{n}\right) u_{n, \lambda}(t)-\mu R\left(\mu, A_{n}\right) u_{n, \mu}(t)\right)> \\
+ & <A_{n, \lambda} u_{n, \lambda}(t)-A_{n, \mu} u_{n, \mu}(t), J\left(\lambda R\left(\lambda, A_{n}\right) u_{n, \lambda}(t)\right. \\
& \left.\quad-\mu R\left(\mu, A_{n}\right) u_{n, \mu}(t)\right)> \\
\leq & \left(\left\|A_{n, \lambda} u_{n, \lambda}(t)\right\|+\left\|A_{n, \mu} u_{n, \mu}(t)\right\|\right) \| J\left(u_{n, \lambda}(t)-u_{n, \mu}(t)\right) \\
& \quad-J\left(\lambda R\left(\lambda, A_{n}\right) u_{n, \lambda}(t)-\mu R\left(\mu, A_{n}\right) u_{n, \mu}(t)\right) \| \\
+ & <A_{n}\left(\lambda R\left(\lambda, A_{n}\right) u_{n, \lambda}(t)-\mu R\left(\mu, A_{n}\right) u_{n, \mu}(t)\right), J\left(\lambda R\left(\lambda, A_{n}\right) u_{n, \lambda}(t)\right. \\
& \left.\quad-\mu R\left(\mu, A_{n}\right) u_{n, \mu}(t)\right)> \\
\leq & 2\left\|A_{n} x\right\| \varepsilon
\end{aligned}
$$

for sufficiently large $\lambda$ and $\mu$. Let $M=\sup _{n \geq 1}\left\|A_{n} x\right\|$. Then we have

$$
\frac{d}{d t}\left\|u_{n, \lambda}(t)-u_{n, \mu}(t)\right\|^{2} \leq 4 M \varepsilon
$$

Integrate this inequality from 0 to $t$, then we have $\left\|u_{n, \lambda}(t)-u_{n, \mu}(t)\right\|^{2}$ $\leq 4 M T \varepsilon$. Letting $\mu \rightarrow \infty$, then $\left\|u_{n, \lambda}(t)-u_{n}(t)\right\|^{2} \leq 4 M T \varepsilon$. Since $\varepsilon$ is arbitrary, we have

$$
\lim _{\lambda \rightarrow \infty}\left\|u_{n, \lambda}(t)-u_{n}(t)\right\|=0,
$$

uniformly with respect to $n$.

It remains to show that

$$
\lim _{n \rightarrow \infty}\left|<e^{t A_{n, \lambda_{0}}} x-e^{t A_{\lambda_{0}}}, \phi>\right|=0
$$

for sufficiently large $\lambda_{0}$.

Since $A_{n, \lambda_{0}} x=\lambda_{0}^{2} R\left(\lambda_{0}, A_{n}\right) x-\lambda_{0} x$ and $w-\lim _{n \rightarrow \infty} R\left(\lambda_{0}, A_{n}\right)^{k} x=$ $R\left(\lambda_{0}, A\right)^{k} x, k=1,2, \cdots,<A_{n, \lambda_{0}}^{k} x, \phi>=<\left(\lambda_{0}^{2} R\left(\lambda_{0}, A_{n}\right)-\lambda_{0}\right)^{k} x, \phi>$ converges to $<\left(\lambda_{0}^{2} R\left(\lambda_{0}, A\right)-\lambda_{0}\right)^{k} x, \phi>=<A_{\lambda_{0}}^{k} x, \phi>$ as $n \rightarrow \infty$. Also since $e^{t A_{n, \lambda_{0}}}=\sum_{k=0}^{\infty} \frac{t^{k}}{k !} A_{n, \lambda_{0}}^{k}$, we have

$$
\lim _{n \rightarrow \infty}<e^{t A_{n, \lambda_{0}}} x-e^{t A_{\lambda_{0}}}, \phi>=0 .
$$

Therefore, we have $w-\lim _{n \rightarrow \infty} T_{n}(t) x=T(t) x$ for $x \in D$.

Let $x \in X$. Since $D$ is dense in $X$, there exist $x_{l}$ in $D$ such that $\lim _{l \rightarrow \infty} x_{l}=x$, Since $\left\{T_{n}(t): t \geq 0\right\}$ and $\{T(t): t \geq 0\}$ are contraction 
$C_{0}$ semigroups,

$$
\begin{aligned}
& \left|<T_{n}(t)-T(t) x, \phi>\right| \\
& \leq \quad\left|<T_{n}(t) x-T_{n}(t) x_{l}, \phi>\right|+\left|<T_{n}(t) x_{l}-T(t) x_{l}, \phi>\right| \\
& \quad+\left|<T(t) x_{l}-T(t) x, \phi>\right| \\
& \quad \leq \quad 2\left\|x_{l}-x\right\|\|\phi\|+\mid<T_{n}(t) x_{l}-T(t) x_{l}, \phi>
\end{aligned}
$$

Choose $x_{l_{0}}$ such that $2\left\|x_{l_{0}}-x\right\|\|\phi\|<\varepsilon / 2$. Then $\mid<T_{n}(t) x_{l_{0}}-$ $T(t) x_{l_{0}}, \phi>\mid<\varepsilon / 2$ for sufficiently large $n$. Therefore

$$
\lim _{n \rightarrow \infty}<T_{n}(t) x-T(t) x, \phi>=0 .
$$

\section{Acknowledgment}

The author is very thankful to the referee for very helpful comments.

\section{References}

[1] T. Eisner, A. Sereny, On the weak analogue of the Trotter-Kato theorem, Taiwanese J. Math., 14 (2010), 1411-1416

[2] K. Engel, R. Nagel, One-Parameter Semigroups for linear evolution equations, Springer, Berlin, 2000

[3] G. Marinoschi, A Trotter-Kato Theorem in the weak topology and an application to a singular perturbed problem, J. Math. Anal. Appl., 386 (2012), 50-60

[4] I. Miyadera, Nonlinear Semigroups, Amer. Math. Soc., Providence, Rhode Island, 1992

[5] A. Pazy, Semigroups of linear operators and applications to partial differential equations, Springer-Verlag, 1983

Department of Mathematics

Seoul Women's University

Seoul 139-774, Korea

E-mail: younglee@swu.ac.kr 